Neue Veröffentlichungen zum Thema Allergie:

\section{WALAmed Allergien und Beratungs- karte Allergische Haut- und Schleim- hauterkrankungen}

Jeder dritte Deutsche leidet inzwischen nach Angaben des Ärzteverbands Deutscher Allergologen (ÄDA) an einer Allergie. Symptome in Form von Ekzemen und Pollinose gehören dabei zu den häufigsten Auswirkungen. Aus diesem Anlass hat die WALA Heilmittel GmbH die Haut zum Jahresthema erklärt, dem u.a. Veröffentlichungen wie das neu erschienene «WALAmed Allergien» und die Beratungskarte «Allergische Haut- und Schleimhauterkrankungen» für Verordner gewidmet sind.

Diese beiden Publikationen sollen die therapeutischen Möglichkeiten der anthroposo- phisch erweiterten Medizin bei der Behandlung allergischer Haut- und Schleimhauterkrankungen aufzeigen. Sie beantworten Fragen rund um Eignung und Anwendung von WALA Präparaten bei den häufigsten Beschwerden, die mit diesen Erkrankungen einhergehen.

Die Schrift «WALAmed Allergien» thematisiert die Behandlung der Pollinose sowie allergischer Dermatosen aus fachärztlicher Sicht und praxisbezogen anhand von Kasuistiken, die von klinischen Bildern begleitet werden. Beide Autorinnen, die Allergologin und Dermatologin Dr. med. Brigitte Roesler sowie die anthroposophische Ärztin Dr. med. Franziska Roemer, können auf langjährige ärztliche Erfahrung zurückblicken, die die Grundlage sowohl für bewährte Therapievorschläge als auch für ihre Sichtweise des menschenkundlichen Hintergrundes bildet. So soll das «WALAmed Allergien» den Verordnern kompetente Unterstützung bei der Behandlung dieses komplexen Krankheitsbildes bieten.

Die Beratungskarte «Allergische Haut- und Schleimhauterkrankungen» gibt hingegen in tabellarischer Form eine Übersicht zu Therapieansätzen mit ausgesuchten WALA Arzneimitteln und beschreibt dabei auch die besonderen Eigenschaften der einzelnen Präparate.

Weitere Informationen bei

WALA Heilmittel GmbH

Presse- und Öffentlichkeitsarbeit

Celia Schönstedt

Boßlerweg 2

73087 Bad Boll/Eckwälden

Tel.+49 7164 930-245, Fax -9245

celia.schoenstedt@wala.de

\section{Auf die Mischung kommt es an:}

\section{Ergänzende Therapie bei Krebs}

Das Leiden fängt für Krebspatienten oft erst an, wenn die eigentliche Therapie längst eingeleitet ist: Sie kämpfen mit Übelkeit, Mattigkeit und häufig auch mit erheblicher Infektionsanfälligkeit. Die lebensnotwendigen Chemo- oder Strahlentherapien fordern ihren Preis. Kein Wunder, dass die meisten Krebspatienten auf eine begleitende Behandlung bauen, die diese Nebenwirkungen unterdrückt oder gar verhindert.

Wichtig für die Erholung von Operationen, Chemo- oder Strahlentherapie und für eine angemessene Lebensqualität sind so genannte Mikronährstoffe: Vitamine, Mineralstoffe und Spurenelemente, denen die Deutsche
Krebshilfe in ihrer Broschüre «Ernährung bei Krebs» «große Wirkung» zuschreibt. Voraussetzung ist allerdings, dass die Patienten sie in der richtigen Dosierung zu sich nehmen. Durch normale Nahrungsmittel ist dies oft nicht zu gewährleisten. Zum einen kann ein Betroffener schwer einschätzen, wie viel Beta-Karotin oder Vitamin A er beispielsweise durch Essen von Gemüse einnimmt. Zum anderen erschweren auch moderne Ernährungsgewohnheiten mit vielen Fertiggerichten die sinnvolle Versorgung mit den lebensnotwendigen Mikronährstoffen. Anders sieht es aus, wenn Patienten oder die Patientinnen auf speziell zusammengestellte Nahrungsergänzungsmittel zurückgreifen, etwa auf CAREIMMUN ${ }^{\circledR}$ Basic. Eine Kapsel enthält eine standardisierte Mischung essentieller Spurenelemente, Vitamine und Mineral- stoffe, die auf die Ernährungssituation von Krebspatienten abgestimmt ist. Dadurch kann der Patient sicher sein, dass er sich diese Mikronährstoffe in gleich bleibender Dosierung und Zusammensetzung zuführt.

Die Deutsche Gesellschaft für Onkologie hat CAREIMMUN ${ }^{\circledR}$ Basic daher als sinnvolle Ergänzung der klassischen Therapie empfohlen. Je besser sich Krebspatienten fühlen und je besser sie ernährt sind, desto förderlicher ist dies für ihre Genesung.

Weitere Informationen bei biosyn Arzneimittel GmbH Presse und Öffentlichkeitsarbeit Schorndorfer Straße 32

70734 Fellbach

Tel.+49 $71157532-35$, Fax -668

presse@biosyn.de

\title{
Ticker+++ Ticker+++ Ticker+++ Ticker+++ Ticker+++ Ticker+++ Ticker+++
}

wandrey. Der Kostendruck auf dem Gesundheitsmarkt führt zu Verdrängungsprozessen, die auch gut eingeführte deutsche Hersteller zwingen, ihre Produkte zu verändern. Im Markt der Akupunkturnadeln, in dem die Produkte kaum veränderbar sind und Qualitätseinbußen hochwertiger Marken nicht akzeptiert würden, geht das nur über die Entwicklung und wirtschaftliche Umsetzung neuer Herstellungsweisen. Mit der neuen stabilo ${ }^{\circledR}$ hat der Berliner Hersteller diesen Schritt erfolgreich getan.

Weitere Informationen bei

Akupunkturnadeln wandrey

Jan Wandrey

info-med@ wandrey.biz
Weleda bietet für Fachkreise eine neue Broschüre: «Weleda Heuschnupfenspray und Gencydo $^{\circledR}$ in der täglichen Praxis». Diese enthält Informationen über Grundlagen der Arzneimittelfindung und über das Krankheitsbild der allergischen Rhinokonjunktivitis. Anhand allgemeiner Richtlinien, aktueller Studienergebnisse und differenzierter Falldarstellungen werden die unterschiedlichen Möglichkeiten der Anwendung der beiden Präparate besprochen.

Kostenfreie Bestellung für Fachkreise

Weleda Kundenbetreuung

Art. Nr. 50708500

Tel. +49 7171 919-109, Fax -200

kundenbetreuung@weleda.de
PASCOE. Schonende Hilfe bei gutartiger VergröBerung der Prostata und bei Beschwerden der Harnblase bieten jetzt die neuen URO-PASC ${ }^{\circledR}$ Tabletten. Als homöopathisches Kombinationsarzneimittel enthalten die Tabletten die folgenden arzneilich wirksamen Bestandteile: Chimaphila umbellata, Pareira brava, Populus tremuloides und Sabal serrulatum. Sonstige Bestandteile sind: Lactose-Monohydrat, Maisstärke und Calciumbehenat.

Weitere Informationen bei

PASCOE pharmazeutische Präparate $\mathrm{GmbH}$ Stefanie Wagner-Suske info@pascoe.de 